\title{
Assessment of Anxiety on Mathematics for Students in Secondary School in Qatar
}

\author{
$\underline{\text { Ali Alzahrani }}^{\text {a }}$ and Elizabeth Stojanovski ${ }^{\text {a }}$ \\ ${ }^{a}$ University of Newcastle, Australia \\ Email: alirashashr.alzahrani@uon.edu.au
}

\begin{abstract}
Various factors including gender, anxiety, parent socio-economic status and school-specific aspects affect mathematics performance. An analysis of the Program for International Student Assessment (PISA) 2012 data on mathematics performance for secondary school students in Qatar was undertaken. This data includes information on student anxiety and gender among other student and school factors that may potentially impact learning outcomes in the form of mathematics test scores. Results indicate a negative statistically significant relationship between mathematics anxiety and mathematics test scores. This inverse relationship was supported after adjustment was made for other factors that may also potentially affect mathematics performance, indicating lower mathematics scores, on average, among anxious students. It is hypothesised that an attributing factor to the observed association between mathematics anxiety and mathematics performance may be the teaching strategies used that may not adequately attend to the cognitive and psychological needs of students. The degree to which teaching strategies implemented attend to the cognitive and psychological needs of students is a potential area that could be targeted to help improve learning outcomes among students studying mathematics at secondary school. This study assesses the efficacy of various teaching approaches and comparatively assesses their impact on mathematic performance in relation to anxiety levels among students. The implications of this research on suggestions for potential changes to current teaching strategies to acknowledge the potential impact that mathematics anxiety has on outcomes in mathematics is discussed. It is proposed that teaching strategies in Qatar could potentially be reviewed with a view to better cater to the needs of students who are quite anxious about studying mathematics, in an attempt to improve the performance of students nationally as, at present, mathematics performance of students in Qatar is considerably lower than global average standards. Although the average mathematics scores were not significantly different between male and female students, it was observed that males were significantly more anxious compared with females.
\end{abstract}

Keywords: $\quad$ Mathematics anxiety, gender, STEM, mathematics education 


\section{INTRODUCTION}

Students have differing social factors, cognitive functioning and academic abilities, all of which can be associated with varying degrees of anxiety towards mathematics. Combinations of these factors could also be attributable to differing levels of associated anxiety when it comes to studying mathematics and related concepts. Social factors, such as the level of support provided by parents and family, could be an additional or related factor attributing to one's perception of mathematics and hence also to associated anxiety when it comes to studying mathematics concepts, particularly in households associated with low socioeconomic status (SES). Students with poor self-esteem and lower levels of motivation to study mathematics could also be more anxious about their performance (Dobson, 2012).

Cognitive functioning ability is an aspect that can be associated with forgetfulness, for example, when it comes to the learning and retainment of learnt mathematics concepts. Furthermore, a lack of understanding of some concepts when first taught, as a result of personal learning difficulties or a lack of ability to focus, particularly for younger students, can contribute to one's anxiety when it comes to the learning of mathematics (Ruff and Boes, 2014). Working memory is considered an element of cognitive ability and has been shown to be a significant predictor of mathematics anxiety, and can be affected by emotional reaction, for example. In particular, it has been shown that emotional reaction can have negative effects on working memory, resulting in increased anxiety among students (Ramirez et al. 2013).

Teaching methods used and teacher traits could also potentially impact on a student's perception of mathematics. Academic factors such as the use of traditional styles of teaching as well as teachers who themselves have associated anxieties with teaching and/or the associated presentation of mathematics content, could also be potentially influencing students' anxieties when it comes to learning mathematics concepts.

Anxiety towards mathematics has been shown to be a significant predictor of mathematics performance in many studies among both male and female students. Some studies have demonstrated girls to be more anxious about mathematics (Kyttala and Bjorn, 2013) and proposed strategies to motivate and encourage female students to learn mathematics by improving the atmosphere in which mathematic classes were taught. In another study, no statistically significant difference in mathematics anxiety was found between male and female students (Zakaria et al. 2012). The authors of this study proposed that teachers can have an important role to play in terms of recognising mathematics anxiety at early stages of schooling and implementing strategies accordingly to try to reduce anxieties among students (Zakaria et al. 2012).

It has been identified that anxiety towards mathematics is an important barrier that limits students undertaking further studies in mathematics at post-secondary level (Tyson et al. 2007; Winkelman, 2009). This is another factor to consider when tackling the global shortage of suitably trained people working in Science, Technology and Mathematics (STEM) areas. To increase the number of students studying in these areas at secondary and tertiary level first requires addressing of associated anxieties towards these subjects. For the present study, the relationship between students' anxiety and mathematics performance at secondary level is examined among students in Qatar.

\section{METHODS}

For the present study, data from 10966 secondary school students in the country of Qatar participating in the 2012 Programme for International Student Assessment (PISA, 2012) were assessed. PISA measures the performance of 15 years old school students in reading, mathematics and science literacy (OECD, 2013). The questionnaire developed by PISA also contains information on mathematics anxiety, among other items. In the Qatar schools, teachers of male students are only male and teachers of female students are only female as education in Qatar is not a co-education system.

The mathematics anxiety subscale comprised five items: worry that it will be difficult, get very tense, get very nervous, worry about getting poor grades, and feel helpless. The possible responses options for each of these questions are presented in four categories: strongly disagree, disagree, agree and strongly agree. These categories were given scores from one to four respectively so that a higher score for each item was associated with a higher degree of anxiety. The average of these items will be considered as the measure of mathematics anxiety whereby higher values are indicative of higher levels of anxiety towards mathematics. The average anxiety measure was also categorised with an average anxiety score less than 1.75 coded to the first category (1); levels of at least 1.75 and below 2.5 to the second category; values of at least 2.5 and below 3.25 were coded into the third category and levels of at least 3.25 and below 4 coded into the last category.

The overall mathematics performance score was made up of four separate subscales: Shape and Space, Change and Relationships, Uncertainty and Quantity. Seven to ten questions were administrated to students from each 
subscale and correct responses added and used to produce the proportion of administered questions that were answered correctly out of the possible 35, representing the mathematics performance score for each student.

The bivariate association of mathematics anxiety and mathematics score was investigated using Spearman's non-parametric correlation. Further analyses were conducted using anxiety on a categorical scale. In the bivariate setting, the effect of mathematics anxiety on a categorical scale on mathematics performance was assessed using the non-parametric Kruskal-Wallis test to compare the median math performance score between the different anxiety levels and the Mann-Whitney U test was used to compare the median mathematics performance score between male and female students.

Adjustment for other potential predictor variables of mathematics performance score, including gender and socio-economic-status (SES), was also investigated. The measure of socio-economic status (SES) was considered as an adjustment variable in the multivariate analyses. This measure was formed from items used in the PISA study (OECD, 2013) with higher values indicative of a higher measures of SES and is made up of items measuring whether the student has a computer and internet connection, the number of cars in the student's household and the number of rooms in the student's house. The creation of this measure has been described elsewhere (Alzahrani et al. 2017) and is measured on a scale from 0 to 3, with a higher number indicative of a higher SES.

Multilevel regression models were used to assess the relationship between mathematics anxiety and mathematics performance by accounting for students being nested within schools while also adjusting for other variables including SES and gender. Multilevel models permit hierarchies in the data, here school level data, recognising the possibility that students within a school are likely to be more highly correlated compared with the correlation between students from different schools. As such, schools were treated in the models as random effects.

\section{RESULTS}

\subsection{Bivariate Assessment of Mathematics Anxiety and Math Score}

The side-by-side boxplot of mathematics scores for each mathematics anxiety category presented in Figure 1 shows a decline in mathematics scores as anxiety level increases. The distributions of the mathematics performance scores were skewed to the right and differed significantly from a normal distribution (Shapiro Wilk test, $p<0.01$ ), violating the assumption of normality of standard parametric tests.

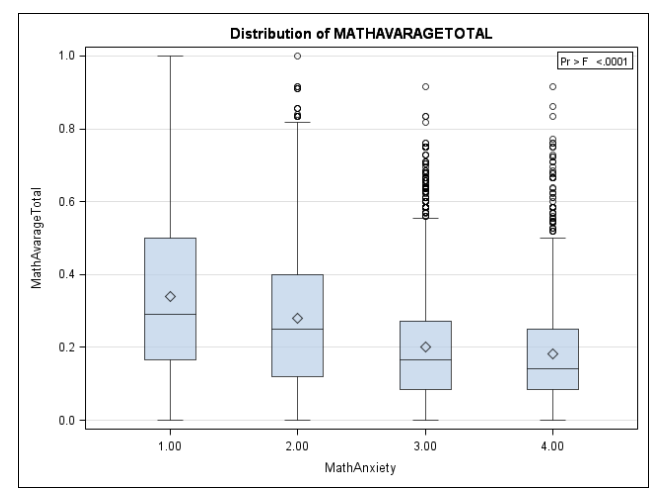

Figure 1. Side by side box-plot of math average score by anxiety level

Bivariate statistical analyses supported the statistically significant difference in median mathematics scores for differing levels of anxiety (Kruskal-Wallis $X^{2}=509, \mathrm{df}=3, p<0.001$ ), with mean ranks of mathematics performance for each math anxiety group supporting the significant negative association between math scores and math anxiety categories. Students who are more anxious appear to have overall lower mathematics performance scores, with all categories of math anxiety significantly different from all other levels of anxiety in terms of the mathematics performance scores (Dunn's post-hoc test, $p<0.01$ ). The negative relationship between anxiety and math performance was also apparent when treating anxiety as continuous (Spearman's correlation coefficient, $\rho$ (rho) $=-0.23, p<0.001)$. 


\subsection{Assessment of confounders on relationship between Mathematics Anxiety and Mathematics Score}

The Wilcoxon signed rank test indicated a statistically significant difference in the rank sums of mathematics scores by gender $\left(X^{2}=73.3, \mathrm{df}=1, p<0.001\right)$, whereby the mean rank mathematics performance scores were significantly higher among females compared to males. The effect of mathematics anxiety on mathematics scores, controlling for gender, remained statistically significant (Cochran-Mantel-Haenszel statistic $p<0.001$ ).

It was also found that the effect of gender on anxiety was statistically significant with males significantly more anxious compared to females $(p<0.01)$. Similarly, the SES measure had a significant effect on mathematics anxiety (Kruskal-Wallis test Chi-square $=36, \mathrm{df}=3, p<0.001$ ) with mean rank anxiety scores significantly highest among students in the highest and lowest SES groups, compared with moderate levels of SES. This could be indicative of those with access to more resources being potentially more aware of the importance of performing well at school, thus posing more anxiety among associated students. Similarly for those in the lowest SES group, this could be indicative of not having the ability or resources to perform well, thus being at a disadvantage, contributing to the increased anxiety for students in this group, compared to those in the middle two categories of SES. The mean rank scores for the lowest to the highest categories (levels 0-3) of the wealth measure (which was an indicator of SES) were 4001, 3415, 3246 and 4052 respectively. Comparing the moderate levels of wealth (levels 1 and 2), it was observed that students with the higher SES level were less anxious compared with the other group. This is also apparent from the side-by-side boxplot in Figure 2. All levels of SES were significantly different from other levels on median anxiety (Dunn's post-hoc test, $p<0.001$ ), except for the difference in anxiety between the highest and lowest SES measures which was not statistically significant $(p>0.05)$.

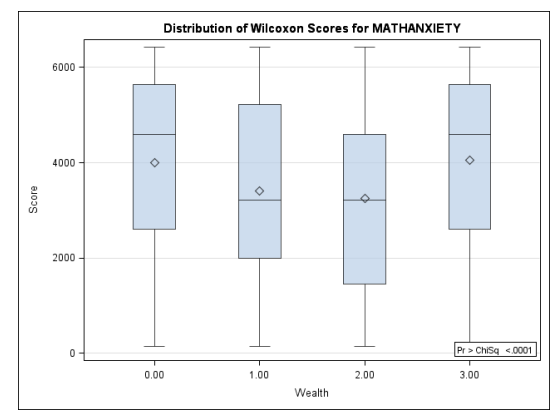

Figure 2. Side-by-side box-plot of anxiety scores by SES levels (indicated by Wealth on the x-axis)

For the final multilevel regression model to account for students being nested within schools for the Qatar data, SES, gender and mathematics score were included as predictors and anxiety score as the response. It was found that male students with high level of wealth (SES) were more anxious compared with other male students. While for low level of wealth (SES), males were more anxious compared with females, indicating that male students are more sensitive to the SES parameter. The random effect of school was statistically significant in the model $(p<0.01)$.

\subsection{Each item of Anxiety by Gender and Mathematics score}

Correlation of the items making up the anxiety subscale with mathematics score indicated consistently significant $(\mathrm{p}<0.001)$ and negative associations. The correlations of four of the items making up the anxiety scale (worry that it will be difficult, get very tense, get very nervous, worry about getting poor grades) with mathematics score were approximately -0.25 while item 'feel helpless' had a slightly lower correlation ( $\mathrm{r}=$ $0.15)$ with mathematics score.

Male and female students did not significantly differ on the following items of math anxiety: get very tense, get very nervous and feel helpless, while males were significantly more anxious than females for the remaining two items $(\mathrm{p}<.001)$ : worry that it will be difficult, worry about getting poor grades, suggesting potentially greater pressure among male students to perform in these areas, although females appeared to score statistically significantly higher on mathematics scores overall.

\subsection{Each Mathematics Subscale by Anxiety and Gender}

The previously identified significant negative correlation between mathematics anxiety and mathematics scores is consistent when each subscale of mathematics is considered separately $(p<0.001$; Spearman's 
correlation coefficient: $\rho$ (rho): Shape and Space: -0.20; Change and Relationships: -0.23 , Uncertainty: -0.23 and Quantity: -0.25).

A side-by-side box plot of mean ranks of Shape and Space scores by mathematics anxiety categories in Figure 3 confirms this negative trend with increasing anxiety levels (Kruskal-Wallis Chi-Square $=27.1092, \mathrm{df}=1, p<$ 0.001). This was consistent with the remaining three subscales of mathematics (Change and Relationships, Uncertainty and Quantity).

The previously identified significant difference in performance on mathematics scores between genders with females performing significantly better than males is also consistent when considering each mathematics subscale separately (Wilcoxon Scores rank sums test, $p<0.001$ ). Furthermore, male students were significantly more anxious than female students on each measure $(p<0.01)$.

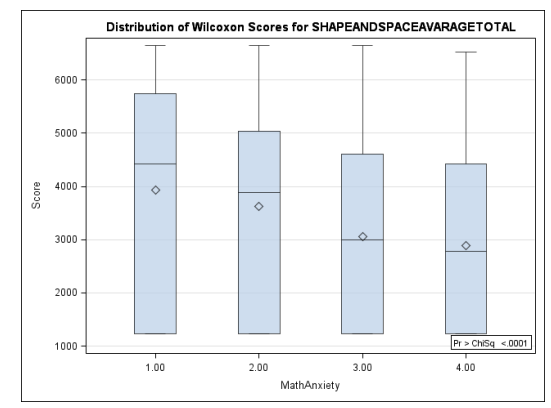

Figure 3. Side-by-side box-plot of Shape and Space mathematics scores by Anxiety level

\section{CONCLUSION}

It appears that performance in mathematics is strongly affected by anxiety towards mathematics. Results support that students who were more anxious about mathematics also performed worse among students in Qatar. Differences in mathematics scores of male and female were significant with males scoring less than females, while males were significantly more anxious than females. This finding was contrary to findings from most studies whereby female students tend to be more anxious than males. Literature suggests that female teachers may have better relationships with students than male teachers, which could be a contributing factor to lower anxiety levels among female students in Qatar as they are taught exclusively by female teachers, and hence could have closer and less conflictual relationships with their teachers compared with male students who are taught exclusively by male teachers (Spilt et al. 2011). This potential interaction could imply that higher anxiety levels among males may be due to the pressure for them to perform better as a factor contributing to the poorer performance in mathematics among males compared with females. This suggests the potential for teaching practices to accommodate dealing with anxiety issues at an early stage of schooling and these findings may also help to identify students who are more likely to be affected. Teaching strategies and approaches could have an important role in identifying students at increased risk of performing poorly and also, more broadly, at using methods that help to alleviate issues of anxiety towards mathematics which could, longer term, help to increase the number of students studying mathematics subjects at tertiary level with the ultimate aim of increasing the number of students taking up careers in STEM areas (such as modelling and simulation), which will help to alleviate the international shortage of suitably trained people in these areas.

\section{REFERENCES}

Alzahrani A., Stojanovski E. and Howley P. (2017). Assessment of Teaching Practices on Mathematics for Students in Qatar, Proceedings of the International Association for Statistics Education (IASE).

Dobson C. (2012). Effects of academic anxiety on the performance of students with and without learning disabilities and how students can cope with anxiety at school. Michigan University, 3.

Kyttala M. and Bjorn P.M. (2013). The role of literacy skills in adolescents' mathematics word performance: controlling for visuo-spatial ability and mathematics anxiety. Learning and Individual Differences, 29(1) 59.

OECD (2013). PISA 2012 Assessment and Analytical Framework: Mathematics, Reading, Science, Problem Solving and Financial Literacy. OECD Publishing, 13-20. 
PISA (2012). Programme for international student Assessment, What Students Know and Can Do: Student Performance in Mathematics, Reading and Science, OECD, 1: 23-564.

Ramirez G., Gunderson E.A., Levine S.C. and Beilock S.L. (2013). Math anxiety, working memory and math achievement in early elementary school. Journal of Cognition and Development, 14(2): 188.

Ruff S.E. and Boes S.R. (2014). The Sum of All Fears: The effects of math anxiety on math achievement in fifth grade students and the implications for school counselors, Georgia School Counselors Association Journal, The University of West Georgia, 21(1), 2.

Spilt J.L., Komeen H.M.Y. and Jak S. (2011). Are boys better off with male and girls with female teachers? A multilevel investigation of measurement invariance and gender match in teacher-student relationship quality. Journal of School Psychology, 50(3) 363.

Tyson W., Lee R., Borman K.M. and Hanson M.A. (2007). Science, technology, engineering, and mathematics (STEM) pathways: High school science and math coursework and postsecondary degree attainment. Journal of Education for Students Placed at Risk. 12(3), 243

Winkelman P. (2009). Perceptions of Mathematics in Engineering. European Journal of Engineering Education. 34(4), 305-316.

Zakaria E., Zain N.M., Ahmad N.A. and Erlina A. (2012). Mathematics anxiety and achievement among secondary school students. American Journal of Applied Sciences, 9(11): 1761. 